\title{
Characterization and Area Estimation of MR Brain Tumor Images
}

\author{
K. Vidya Sagar \\ Deptt of ECE, SSIT, \\ Sathupally,
}

\author{
A.Bhujanga Rao \\ Instrument Technology \\ Andhra University,Vsp.
}

\author{
T.Madhu \\ Swarnandra Institute of Engg. \\ \& Tech, Narsapur
}

\begin{abstract}
Brain Tumors are detected efficiently by using the Magnetic Resonance Imaging (MRI) techniques. The extracted MRI image is sensitive to noise which limits the visibility of certain characteristics of the image. This unwanted noise can be disintegrated and curtailed from the original Image by using the non linear digital median filter. The intensification is achieved by using the combination of discrete wavelet transform (DWT) and stationary wavelet transform (SWT) augmented with Bi-cubic interpolation algorithm. The quality of the resolute image is analyzed with the possible static parameters. Segmentation and clustering approaches are applied on the resultant sharpened high resolution image to estimate the tumor area. The results obtained with the proposed Optimized Hybrid Clustering (OHC) algorithm are compared with the existing K-Means, Pillar K-Means and Fuzzy C-means (FCM) approaches. The tumor sensitivity and accuracy of the test parameters with the OHC approach are estimated. In-order to deliver the drug to kill the aberrant tissue, the number of tumor cells is estimated against its radius.
\end{abstract}

\section{Keywords}

Denoise, DWT, SWT, Bi-cubic Interpolation, Segmentation, Tumor area

\section{INTRODUCTION}

The cells in a human brain will develop in a controlled manner. The old and flawed cells will be recovered with new cells. The cell division grows in undisciplined manner and forms as an aberrant tissue labeled as Tumor. Tumors are classified into primary and secondary tumors. Treatment of the tumors depends on its type, area and location. Magnetic Resonance Imaging (MRI) is adopted as reliable and superior approach to trace the brain tumors. MRI Scan is a non invasive procedure that uses strong magnetic field of 21.1 Tesla augmented with radio frequency waves as pulses to attain the images of the brain. Three gradient magnets X, Y\& $\mathrm{Z}$, each one's field aligned at different plane of the organ, are used in MRI. The gradient magnetic field focuses on the distinct area of the slice and work in association with radio frequency pulses to produce the image. The sequence of $\mathrm{X}, \mathrm{Y}$, $\mathrm{Z}$ gradients is used to generate image of the slices in any orientation. The MRI image consists of Grey and white pixels. The grey pixels represent both signal intensity and noise. By increasing the electrical current in the wires of the gradient magnetic field the main magnetic field opposes it and generates huge noise. The Noisy image may introduce inadequate allotment of Gray level pixels as white level pixels and as a result the visibility of certain characteristics of the image gets minimized. However, the visibility of low contrast MRI images can be improved by removing the noise with a median filter. A Non linear digital median filter is used to remove the noise in MRI images and to safeguard the edges of the image. Each pixel in the image relates with nearby neighbours to determine the representative of its urroundings. The median of neibouring pixel value is determined to modify the pixel values as the median is more potent average than mean. Further, the median value will not be affected with aberrant single pixel in a neighbourhood. This approach saves the sharp edges in MRI brain tumor images as the filter does not generate improbable pixel values. Substantial noise is withdrawn from the MRI brain image with median filter [1][2]. The de noised image is further enhanced using combination of discrete wavelet transform (DWT) and stationary wavelet transform (SWT) with applied bicubic interpolation approach to generate sharp high resolution images. The high frequency components of the MRI image are perpetuated using stationary wavelet transform method. The loss of report arises by virtue of down sampling in discrete wavelet transform (DWT) begins in the respective sub bands. SWT is used to curtail the information loss. The discrete stationary wavelet transform disintegrates the MRI image into low and high frequency sub bands [3][4][5][6]. Bicubic interpolation is then applied to sub band images by using nearest neighbour image resampling. The re-sampled images are stable. The proposed SWT with Bicubic interpolation method generates sharper high resolution MRI image [7]. The enhanced high resolution MRI image quality is analyzed with static parameters. The quantitative analysis concludes that the combination of DWT+SWT with applied bi-cubic interpolation results in reliable resolute image. Which was further processed using segmentation and clustering algorithms. The Optimized hybrid clustering $(\mathrm{OHC})$ algorithm is enforced to cluster the tumor associated pixels. These pixel values are used as optimum values to estimate the area of the tumor from MRI images.

\section{TECHNICAL REVIEW}

\subsection{De-noising}

Transform domain and spatial filtering approaches are adopted for MRI images. The compelling noise in MRI images is curtailed by adopting spatial filters. Mean and wiener filters are linear and median filter is nonlinear. The salt and pepper noise in MRI images is curtailed with non linear digital median filter. While removing the noise the filter safeguards the edges. An array of MRI Pixel points are glanced to elect the representative of its surroundings. The median of neighboring pixel value is to be determined to refine each pixel value of the MRI image. The median filter is alleged to be edge secure. The estimated median value will substitute the pixel examined with the center pixel value. If the considered neighbourhood has an even number of pixel points, the average of the two centre pixels is used.

$\mathrm{T}(\mathrm{x}, \mathrm{y})=\mathrm{I}\left(\frac{m \times n}{2}\right)$

Eq1 
$\mathrm{I}_{1} \leq \mathrm{I}_{2} \leq \mathrm{I}_{3} \leq \cdots \leq \mathrm{I}_{\mathrm{n} \times \mathrm{n}}$

Eq2

Where, $\mathrm{T}$ is the threshold value and $I(n \times n / 2)$ is the centre pixel intensity value. As edges contain important information for segmentation, median filter is used for preserving significant details in MRI image. Using wiener filter compelling noise in an image is to be diminished by correlate with desired noise free image. Mean wiener and median filters are used for comparative study of MRI brain tumor images. Wiener filters update the pixel value by averaging of its neighborhoods. . Mean wiener and median filters are used for comparative study of MRI brain tumor images. The Maximum signal power to the maximum signal noise is indicated as peak signal-to-noise ratio (PSNR). The PSNR is determined using the following equation

PSNR $\mathrm{db}=10$ Log $102552 / \mathrm{MSE}$

Where, $M S E=\sum_{x=0}^{M-1} \sum_{y=0}^{N-1}\left[I(x, y)-I^{y}(x, y)\right]^{2}$

Where, $\mathrm{M}=$ Number of rows in an image

$\mathrm{N}=$ Number of columns in an image

MSE is mean squared error, $\mathrm{I}$ is an input image and $\mathrm{I}$ is output image [1][2].

\subsection{Image intensification}

Discrete wavelet transform and Stationary wavelet transform are used to resolute the Low resolution MRI input image. The information loss on its edges i.e high frequency components may arise by applying the interpolation for image intensification. Safeguard the edges is imperative. DWT has been hired to safeguard the high frequency components of the image. In-order to obtain the redundancy and shift invariance the discrete wavelet transform coefficients are essentially interpolated.

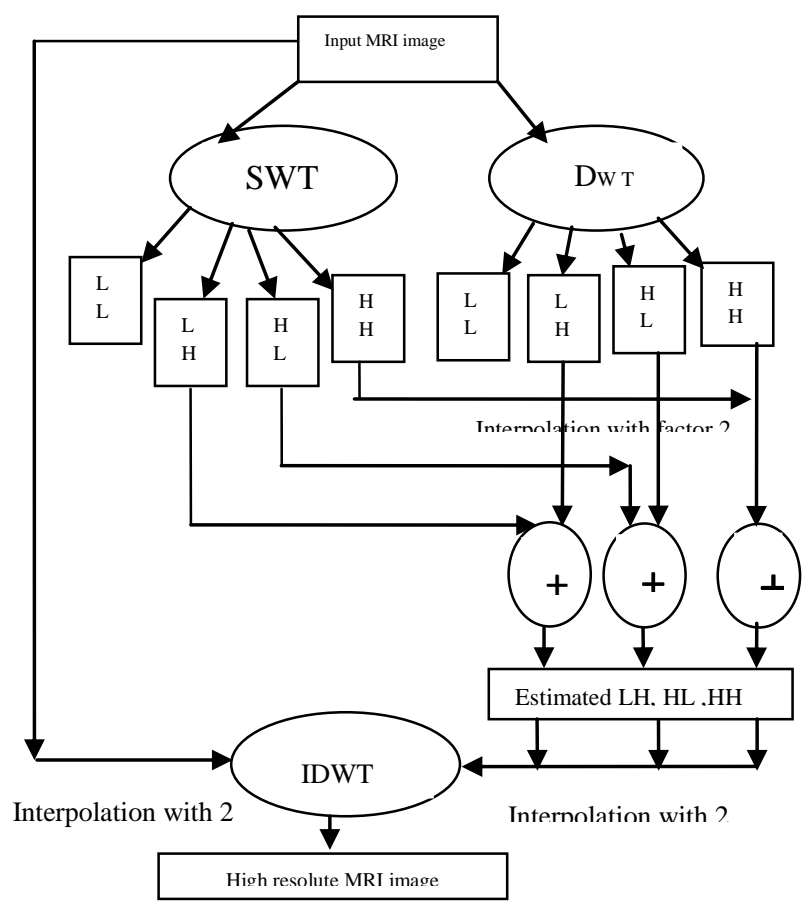

Fig.1 Block diagram of image intensification

Hence DWT is used to disintegrate the MRI image into sub band images. The high frequency components of the image labeled as sub bands LH, HL, HH. For interpolating data points on a two dimensional regular grid, $\mathrm{Bi}$ cubic interpolation is used in order to obtain smooth surface. A growth factor of ' 2 ' for Bi cubic interpolation is enforced on high frequency sub band images. Stationary wavelet transform (SWT) is adapted to negotiate the information loss in the Sub bands due to down sampling. The MRI brain tumor image disintegrates into different sub band images low-low (LL)), low-high (LH), high-low (HL), and high-high (HH). The SWT High frequency sub bands and interpolated high frequency sub bands are combined with each other. Further intensification is achieved by interpolating the estimated high frequency sub bands. The authentic high resolute image is having admirable information than low frequency sub bands. Hence interpolation with factor 2 is applied on the input low resolution MRI image. Inverse discrete wavelet transform (IDWT) is applied on the interpolated input image and interpolated high frequency sub bands to achieve super resolute image [5][6]. The quality of the resolute image is analyzed with the possible static parameters

a). Structural Similarity index measure (SSIM)

The pixel intensities from the local patterns of the resolute image are compared with the input image. The value of SSIM represents either ' 0 ' (labeled as zero correlation) or ' 1 ' (labeled as exact correlation). Significant change in structural information is considered as degradation of the image quality.

\section{b). Laplacian mean squared error (LMSE)}

The laplacian value is estimated by subtracting each Pixel value of the resolute image from the average of neighbouring pixels. The mean square error is estimated based on the laplacian value. The laplacian mean squared error is estimated by considering the ratio of laplacian values of the resolute image and the input image. The large value of 'LMSE' results in poor quality.

\section{c). Structural content (SC)}

The ratio of sum of the pixel values of the original image and the resolute image is quantified to correlate the two images. The Structural content (SC) depends on the similarity between the two images. The similarity measurement is based on low level structural information compared with input image and resolute image.

\section{d). Normalized cross correlation (NCC):}

Due to exposure conditions the brightness of the image may vary and hence the image needs to be normalized. This can be achieved by subtracting the template image $t(x, y)$ with sub image $\mathrm{f}(\mathrm{x}, \mathrm{y})$ and then dividing with the standard deviation.

\section{e). Maximum Difference (MD):}

The large pixel value of the image will be compared with small pixel value. The fine quality of the output image is based on the lowest value of the maximum difference.

\section{f). Normalized Absolute Error ( NAE):}

The numerical difference between the original and the resolute image is considered. The lowest value of NAE represents the fine quality of the image.

\subsection{Segmentation and Clustering}

Image segmentation and clustering practice are imported to estimate the area of the tumor. Image segmentation is classified into Pixel based, regional and edge based methods. Here, the brain tumor images are sub divided into multiple segments as sets of pixels using pixel based segmentation. 
The MRI image incorporates white and grey color pixel elements. White color pixel data points are related to tumor cells and the Gray color pixel data points relate to normal cells. Collection of data point of the pixels that belongs to the same color will be quantified using Euclidian distance method. The clusters may contain large number of pixels. The pixels may be either close or far from the cluster centre. The cluster centre is the mean of the pixel points allocated to that cluster. "Optimized hybrid clustering algorithm" is applied on the Input MRI images to estimate the area of the tumor.

\section{a) Feature Extraction}

A Binary Mask is applied on the MRI input image. Grey and white pixels become brightened. A threshold value is defined to compare with the coefficients of each pixel. If the value lies within the threshold value, a 'Zero' is assigned to that coefficient else a 'One' is assigned. The Fuzzy C Means output is the extracted tumor cluster from the MRI image. The magnitude of the coefficients from the extracted tumor cluster is above the threshold value. The threshold value is represented as T= Max [f(nk) ] + Min $[f(n k)] /$ Avg of $f(n k)$

The MRI input image is represented as ' $\mathrm{f}$ '. Grey level pixels are represented with ' $k$ '. Each pixel in an image ' $\mathrm{f}$ ' is compared with the threshold value $\mathrm{T}$. The threshold value lies in the grey scale range of ' $\mathrm{K}$ '. In-order to define a value to the specific pixel from the output image ' $\mathrm{g}$ ' a binary decision is applied.[7][8][9].

$$
\begin{gathered}
\mathrm{g}(\mathrm{n})=\text { ' } 0 \text { ' if } \mathrm{f}(\mathrm{n}) \geq \mathrm{T} \\
=\text { ' } 1 \text { ' if } \mathrm{f}(\mathrm{n})<\mathrm{T}
\end{gathered}
$$

b) Approximate Reasoning

Linearization method is adopted to estimate the area of the tumor. The MRI image comprises of two pixel values either grey or white ( 0 or 1$)$. The size of the image is considered to be $256 \times 256$. The aggregate of white and black pixels represents the Binary image. [7][8][9].

$I=\sum_{x=0}^{255} \sum_{y=0}^{255}[f(0)+f(1)]$

Pixels $=$ Width $*$ Height $=256 * 256$

Total White Pixals $\left(W_{0}\right)=\sum_{x=0}^{255} \sum_{y=0}^{255} f(1)$

$\mathrm{f}(0)=$ black pixel ' 0 ' $\mathrm{f}(1)=$ White pixel ' 1 '

Where, ${ }^{W_{p}}=$ number of white pixels

1 Pixel value is equated to $0.264 \mathrm{~mm}$

The tumor area is estimated by using

Tumor Size $(S)=\left(\sqrt{W_{p}} * 0.264\right)$

\subsection{Existing methods}

Manual segmentation is commonly time consuming. The error rate is lofty due to imprudent approach. Because of human weariness errors will develop. In accurate boundary determination also will expand the errors. Considering the results progression of significant number of miss detected and false detected pixels causes to produce in accurate tumor area.

In Fuzzy C-Means (FCM), Based on the similarity decomposition and centroids of clusters, determining the membership function is the significant problem. Specifically, determining the number of the cluster is a considerable limitation with FCM. Since the regions are spatially discontinuous grey level similarity is only verified. Considering the experimental study FCM is converging to local minima of the squared error criterion [10].

The k-Means is limited to produce only hyper spherical clusters. It depends on initial centroids. To update the new centroids the mean of the pixel values of the respective clusters need to be estimated. The floating values obtained in some iteration are not favorable. Significantly, positive integers or scalars are required to replace the new centroids. Hence with K-means algorithm optimal solution is difficult to achieve [11] [12].

In pillar k-means the number of clusters will be selected optimally and also the centroid updation for number of iteration will also be solved in pillar k-means by considering the maximum pixel value instead of calculating the mean of group of clustered pixel values [13]

Hence, for a better diagnosis of brain tumor and to improve the performance analysis of clustering algorithms in terms of location finding, running time we proposed a novel method called "optimized Hybrid clustering algorithm".

\section{PROPOSED METHODOLOGY}

Optimized Hybrid Clustering (OHC) algorithm is a combination of pillar k-means and Fuzzy $\mathrm{C}$ means approaches. The combination of these algorithms favorably reduces the difficulties that occurred in previous segmenting algorithms. The membership values $-1,0$, and 1 are applied to the pixels of the cluster centroids, cause to minimize grey level similarity limitation with FCM alone. The floating points obtained with K-Means due to mean calculation for new centroid updating in some iterations has been favorably curtailed by considering the maximum data set point value. The OHC Algorithm substantiates the implication of MRI brain tumor image segmentation and clustering to estimate the tumor area. This approach endorsed optimized results.

\subsection{Optimized Hybrid Clustering Algorithm}

Step1. Normalize the Input image into $256 * 256$ sizes.

Step2. Convert the image into double precision values.

Step3. Reshape the image into data sets of column vector for segmentation.

Step4. Determine the number of clusters i.e. centroids.

Step5. Calculate the distance between input data set points and set points of the centroids.

Step6. Find the data set points which are neighboring to the centroid.

Step7. Select the centroid with minimum distance then move the data set points to the closest relevant centroids

Step8. Re compute the centroids by selecting the maximum pixel value from the set of relevant centroid data points. 
Step9. Repeat the process until the new centroids and the previous centroids are symmetrical.

Step10. Allocate the membership values $-1,0$ and 1 to each pixel of the cluster centroids

Step11. Estimate the global threshold value T.

Step12. Determine the data set points with similar membership value and then reform the new clusters.

Step13.Apply the binary decision to obtain the resultant cluster image $g(n)$

Step14. Estimate the square of number of white pixels $\left(\mathrm{W}_{\mathrm{p}}\right)$

Step15. Compute the area of the tumor "S" using linearization method

\section{RESULTS \& ANALYSIS}

An innovative procedures based on Median Filter, wiener filter and mean filter algorithms are adopted to effectively de noise the MRI brain tumor images and for preserving the edges. While preserving high quality of restored image the noise abolition across an ample range of noise quantity about 30 to $98 \%$ is considered. This is mainly due to the use of median filter for the MRI images. Considering significant number of MRI brain tumor images, Wiener filter deteriorated to preserve the fine point pixel values and edges of the MRI image. The PSNR estimated values for the above three filters are tabulated in Table 1 and shown in Fig.6. The median filter applied to different MRI images caters satisfying results.

The DWT and SWT are used augmented with Bicubic interpolation to enhance the denoised tumor image. Considering fig 2(c), fig 3(c), fig4(c), fig.5(c) introducing the SWT, information loss due to down sampling in DWT is considerably reduced. DWT has been selected to preserve the high frequency components of the MRI image. The image size of $256 \times 256$ is used for enhancement with an interpolation factor of 2. Table 4 and fig7 summarizes the quality of the resolute images. The laplacian mean square error Results less than 1.2 for the tested samples. The normalized absolute error results ' 0.5 '. Less than 1 considered as a best value. The maximum difference and average difference values are approximately close to zero. The peak signal to noise ratio is also favorably good for all the tested samples. Low level structural information i.e within 3.0 when compared with input image and resolute image. Table 4 concludes that SSIM values for all the tested samples are 0.99. I.e. 99 percent of the exact correlation is achieved. Table 2 summarizes the estimated Area of the tumor for all the 4 samples. Manual segmentation and Fuzzy C-Means procedures produce unfavorable results. Pillar K-means produced the tumor area for sample 2 as $14.7013 \mathrm{~mm} 2$. But, the proposed "optimized hybrid clustering approach" proved itself as reliable result for sample 2 with $10.9679 \mathrm{~mm} 2$. Table 2 and fig 7 conclude that the OHC approach produces the optimum tumor area for all the tested samples. While comparing the fig.2(d), 3(d), 4(d) and 5(d) The OHC approach produced the output exactly matched with the actual tumor shape. The total white pixels are precisely detected inorder to efficiently estimate the true area of the tumor. This approach achieved the precision level of about $98 \%$ closest to the true tumor size. The CPU Computation time is also tabulated in Table 3. Even though the computation time of the proposed algorithm is considerably increased owing to the membership values assigned to each pixel values, the iterations are increased to produce precise readings. The tumor sensitivity and accuracy of the resultant image are tabulated. The definite positive tumors cells (white data set points) are decently diagnosed and are measured as sensitivity. Based on in-Vivo and in Vitro calculations if the tumors are considered as a sphere, $1 \mathrm{~cm}$ in diameter of the tumor contains 109 tumor cells. Inorder to deliver the drug to kill the tumor cells an absolute number of cells need to be estimated. Table. 6 and fig.9 shows the relationship between the radius ' $r$ ' and the number of tumor cells.

\section{EXPERIMENTAL RESULTS}

Experiments conducted on primary brain tumor MRI images show that the median filter produced favorable PSNR value and justified as an optimum de-noising method for MRI images.

\section{Tested Results}

\section{Sample1.}
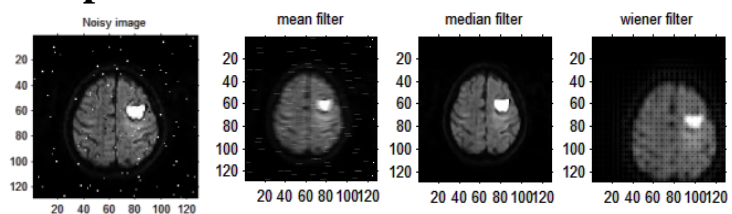

Fig.2(a) Noisy image Fig.2(b) De noised images
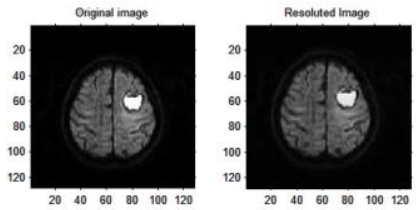

Fig.2(c) Resolute image
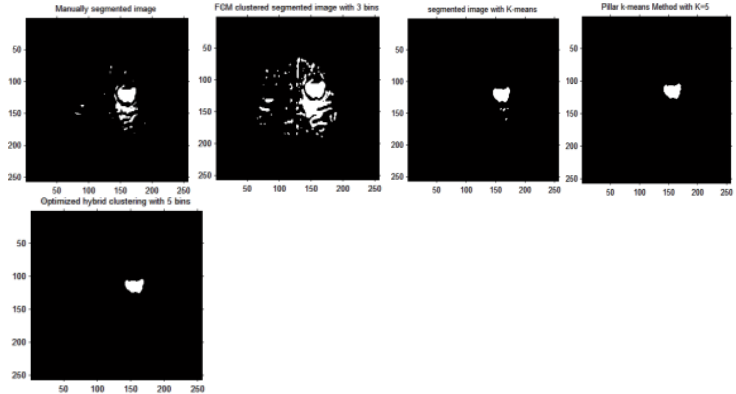

Fig.2 (d) Segmented and clustered image results for sample 1

SAMPLE2:
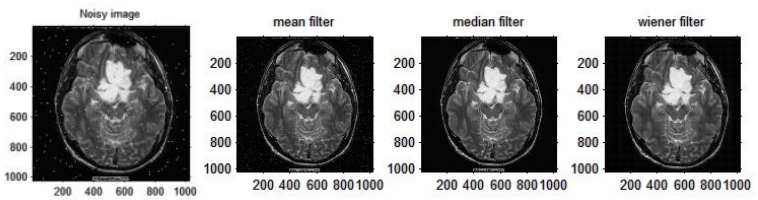

Fig 3(a) Noisy image Fig.3(b) De noised images
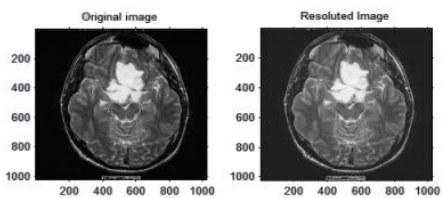

Fig 3(c)Resolute image 

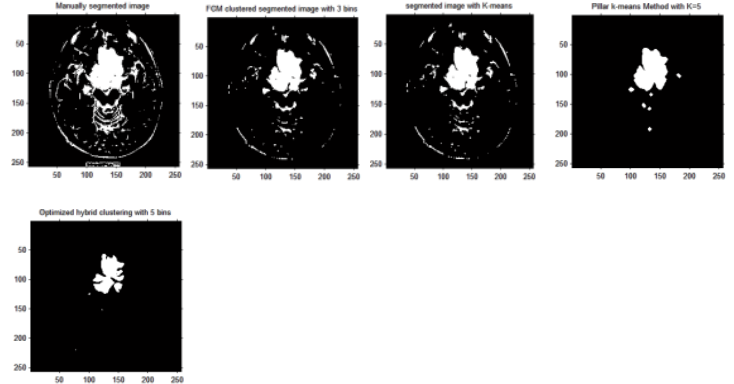

Fig 3(d) Segmented and clustered image results for sample
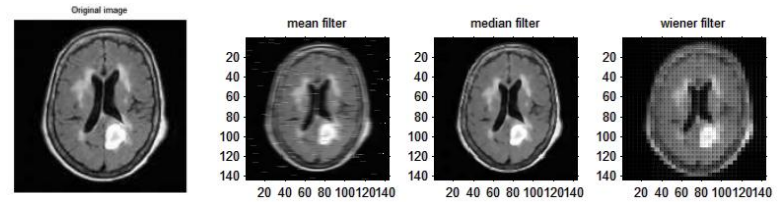

Fig4 (a) Noisy Image Fig 4 (b) De noised image
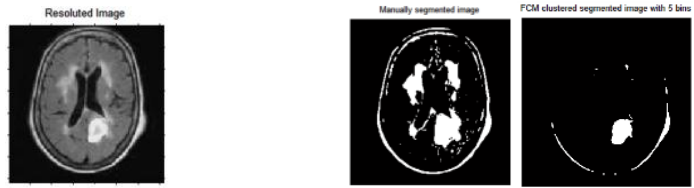

Fig.4(c) Resolute image

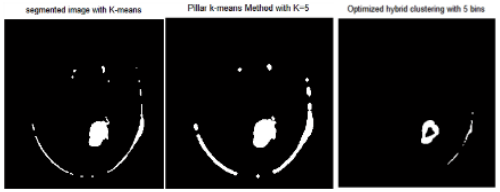

Fig4 (d) Segmented and clustered image results for sample

Sample 4
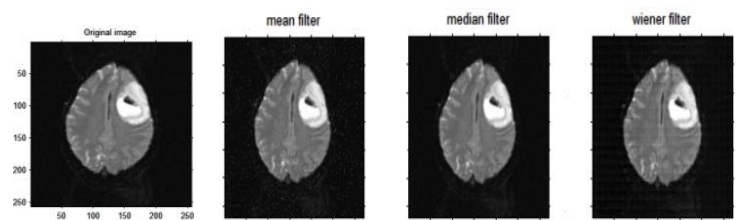

Fig 5(a) Noisy image Fig 5(b) De noised image

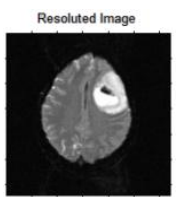

Fig.5(c) Resolute image

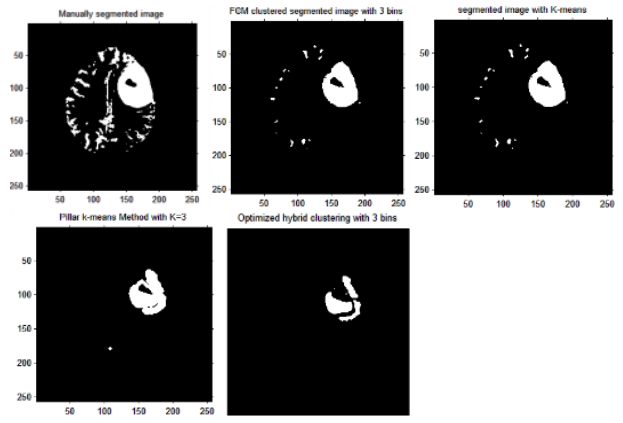

Fig5 (d) Segmented and clustered image results for sample 4
Table 1 PSNR values for various filters.

\begin{tabular}{|c|c|c|c|c|c|}
\hline S.No & \multirow{2}{*}{$\begin{array}{c}\text { Filter } \\
\text { Type }\end{array}$} & \multicolumn{4}{|c|}{} \\
\cline { 3 - 6 } & & Sample1 & Sample2 & Sample3 & Sample4 \\
\hline $\mathbf{1}$ & Average & 47.99 & 50.77 & 42.9404 & 55.0095 \\
\hline $\mathbf{2}$ & Weiner & 38.34 & 41.93 & 40.4671 & 48.4837 \\
\hline $\mathbf{3}$ & Median & 53.17 & 57.75 & 46.5094 & 70.8913 \\
\hline
\end{tabular}

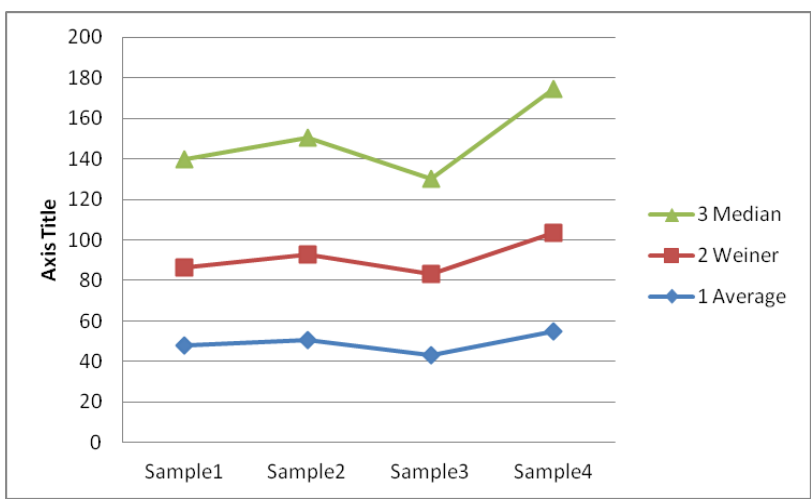

Fig.6 Comparison of PSNR values for various filters

Table.2 Area estimation Comparison for various algorithms

\begin{tabular}{|c|l|c|c|c|c|}
\hline \multirow{2}{*}{ S.No } & \multirow{2}{*}{ Algorithm } & \multicolumn{4}{|c|}{ Area of the tumor( mm^2 ) } \\
\cline { 3 - 6 } & & $\begin{array}{c}\text { Sample } \\
1\end{array}$ & Sample2 & Sample3 & Sample4 \\
\hline 1 & Manual & 7.7914 & 23.2185 & 22.6656 & 18.8829 \\
\hline 2 & $\begin{array}{l}\text { Fuzzy C- } \\
\text { Means }\end{array}$ & $\begin{array}{c}14.627 \\
6\end{array}$ & 17.6998 & 10.8722 & 13.4899 \\
\hline 3 & K-Means & 6.4451 & 18.3304 & 11.1569 & 13.5363 \\
\hline 4 & $\begin{array}{l}\text { Pillar K- } \\
\text { Means }\end{array}$ & 5.9853 & 14.7013 & 14.0442 & 12.3602 \\
\hline 5 & OHC & 5.4935 & 10.9679 & 7.2636 & 9.3971 \\
\hline
\end{tabular}

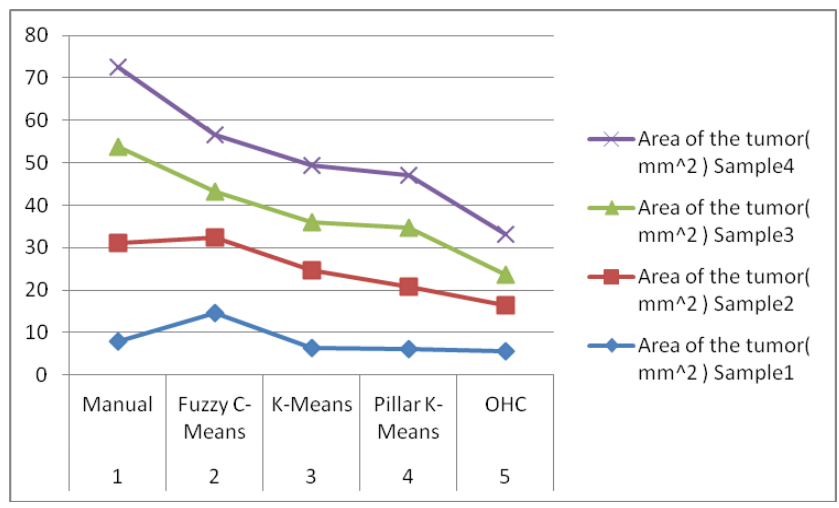

Fig.7 Comparison of Area of the tumors with various approaches 
Table.3 Comparison of CPU Computation time for various algorithms

\begin{tabular}{|c|c|c|c|c|c|}
\hline \multirow{2}{*}{$\begin{array}{c}\text { S.N } \\
\text { o }\end{array}$} & \multirow{2}{*}{ Algorithm } & \multicolumn{4}{|c|}{ CPU Computation time (Seconds) } \\
\cline { 3 - 6 } & Sample1 & Sample2 & Sample3 & Sample4 \\
\hline 2 & $\begin{array}{c}\text { Fuzzy C- } \\
\text { Means }\end{array}$ & 5.6004 & 2.0436 & 20.7793 & 1.0296 \\
\hline 3 & K-Means & 748 & 0.6708 & 1.1700 & 0.6084 \\
\hline 4 & $\begin{array}{c}\text { Pillar K- } \\
\text { Means }\end{array}$ & 0.8580 & 0.6552 & 0.7488 & 0.7644 \\
\hline 5 & OHC & 2.7144 & 5.772 & 11.1697 & 5.4912 \\
\hline
\end{tabular}

Table4. Quality analysis comparison for various sample images

\begin{tabular}{|c|c|c|c|c|c|c|c|c|}
\hline $\begin{array}{c}\text { Sample } \\
\text { No }\end{array}$ & $\begin{array}{c}\text { PS } \\
\text { NR } \\
\text { (db } \\
\text { ) }\end{array}$ & $\mathrm{AD}$ & $\mathrm{SC}$ & $\begin{array}{c}\mathrm{NC} \\
\mathrm{C}\end{array}$ & MD & $\begin{array}{c}\mathrm{L} \\
\mathrm{MS} \\
\mathrm{E}\end{array}$ & $\begin{array}{c}\text { NA } \\
\text { E }\end{array}$ & $\begin{array}{c}\text { SSI } \\
\mathrm{M}\end{array}$ \\
\hline $\begin{array}{c}\text { Sample } \\
1\end{array}$ & $\begin{array}{c}65 . \\
00 \\
78 \\
4 \\
\end{array}$ & $\begin{array}{l}0.04 \\
021\end{array}$ & $\begin{array}{l}3.64 \\
4875\end{array}$ & $\begin{array}{l}0.46 \\
8780\end{array}$ & $\begin{array}{l}0.00 \\
0000\end{array}$ & $\begin{array}{c}1.1 \\
68 \\
93 \\
2 \\
\end{array}$ & $\begin{array}{l}0.56 \\
4376\end{array}$ & $\begin{array}{l}0.9 \\
98 \\
42\end{array}$ \\
\hline $\begin{array}{c}\text { Sample } \\
2\end{array}$ & $\begin{array}{l}69 . \\
20 \\
29 \\
03 \\
\end{array}$ & $\begin{array}{l}0.09 \\
785\end{array}$ & $\begin{array}{l}3.95 \\
6706\end{array}$ & $\begin{array}{l}0.49 \\
4208\end{array}$ & $\begin{array}{r}0.00 \\
7843\end{array}$ & $\begin{array}{c}0.8 \\
99 \\
74 \\
8 \\
\end{array}$ & $\begin{array}{l}0.50 \\
7452\end{array}$ & $\begin{array}{l}0.9 \\
96 \\
85\end{array}$ \\
\hline $\begin{array}{c}\text { Sample } \\
3\end{array}$ & $\begin{array}{l}64 . \\
43 \\
86 \\
26 \\
\end{array}$ & $\begin{array}{l}0.08 \\
832\end{array}$ & $\begin{array}{l}0.08 \\
8326\end{array}$ & $\begin{array}{l}0.50 \\
1255\end{array}$ & $\begin{array}{r}0.02 \\
3492\end{array}$ & $\begin{array}{c}0.5 \\
28 \\
86 \\
6 \\
\end{array}$ & $\begin{array}{l}0.49 \\
6930\end{array}$ & $\begin{array}{l}0.9 \\
95 \\
05\end{array}$ \\
\hline $\begin{array}{c}\text { Sample } \\
4\end{array}$ & $\begin{array}{l}72 . \\
45 \\
00 \\
31\end{array}$ & $\begin{array}{l}0.10 \\
177\end{array}$ & $\begin{array}{l}3.68 \\
2151\end{array}$ & $\begin{array}{l}0.46 \\
6257\end{array}$ & $\begin{array}{r}0.00 \\
3713\end{array}$ & $\begin{array}{c}1.1 \\
19 \\
04 \\
0\end{array}$ & $\begin{array}{l}0.56 \\
6200\end{array}$ & $\begin{array}{l}0.9 \\
97 \\
05\end{array}$ \\
\hline
\end{tabular}

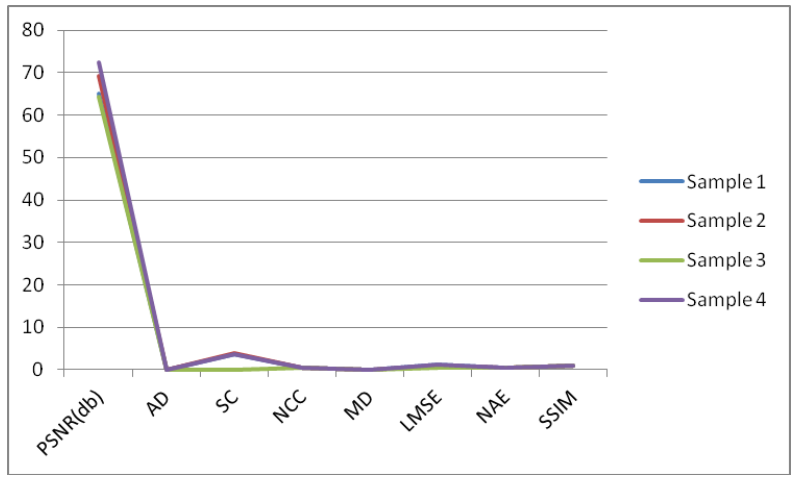

Fig.8 Comparison of Quality parameters for resolute sample images

Table5. Sensitivity and accuracy calculations for various samples

\begin{tabular}{|c|c|c|}
\hline Sample No & Sensitivity & Accuracy \\
\hline Sample 1 & 0.457 & 0.9992 \\
\hline Sample 2 & 0.582 & 0.9500 \\
\hline Sample 3 & 0.697 & 0.9609 \\
\hline Sample 4 & 0.587 & 0.9641 \\
\hline
\end{tabular}
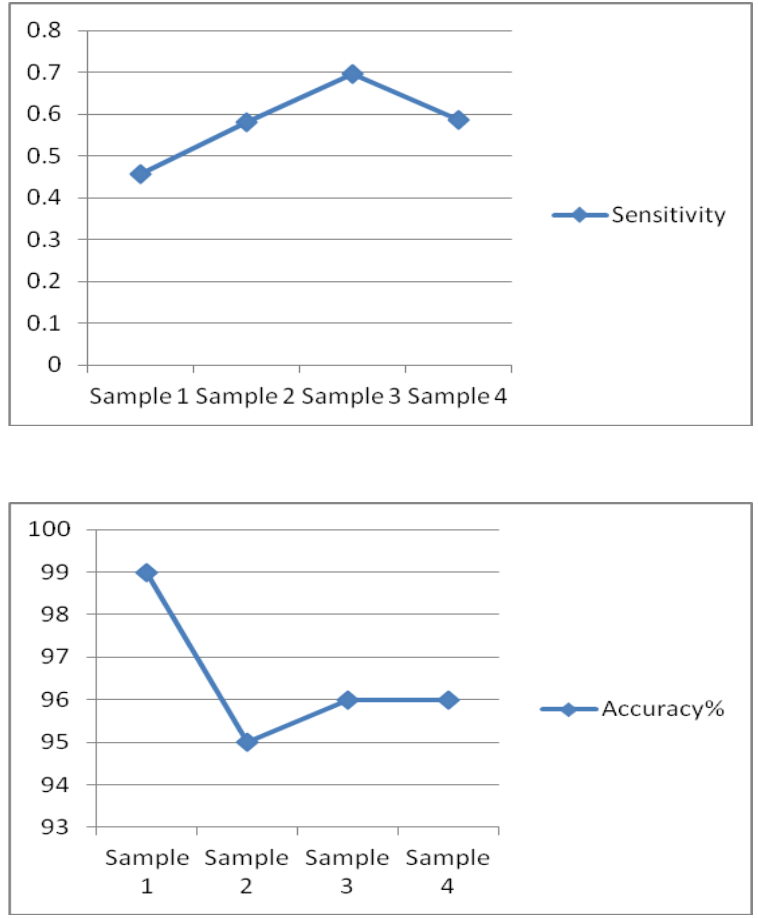

Fig.9 Comparison of medical parameters for various sample images

Table.6 Comparison of tumor cells with its radius and volume

\begin{tabular}{|c|c|c|c|c|}
\hline $\begin{array}{c}\text { Sample } \\
\text { No }\end{array}$ & $\begin{array}{c}\text { Radius } \\
(\mathrm{cm})\end{array}$ & $\begin{array}{c}\text { Volume } \\
\left(\mathrm{m}^{3}\right)\end{array}$ & $\begin{array}{c}\text { Number } \\
\text { of cells } \\
(\mathrm{N}) * 10^{9}\end{array}$ & $\begin{array}{c}\text { Uni- } \\
\text { dimen } \\
\text { sional } \\
\text { diamet } \\
\text { er (2r })\end{array}$ \\
\hline Sample 1 & 0.13 & $\begin{array}{c}0.219 * 10^{-} \\
8\end{array}$ & $0.26 * 10^{9}$ & 0.26 \\
\hline Sample 2 & 0.18 & $\begin{array}{c}0.243^{*} 10^{-} \\
7\end{array}$ & $0.36 * 10^{9}$ & 0.36 \\
\hline Sample 3 & 0.15 & $\begin{array}{c}0.1405 * 10 \\
-7\end{array}$ & $0.3 * 10^{9}$ & 0.3 \\
\hline Sample 4 & 0.17 & $\begin{array}{c}0.2047 * 10 \\
-7\end{array}$ & $0.34 * 10^{9}$ & 0.34 \\
\hline
\end{tabular}

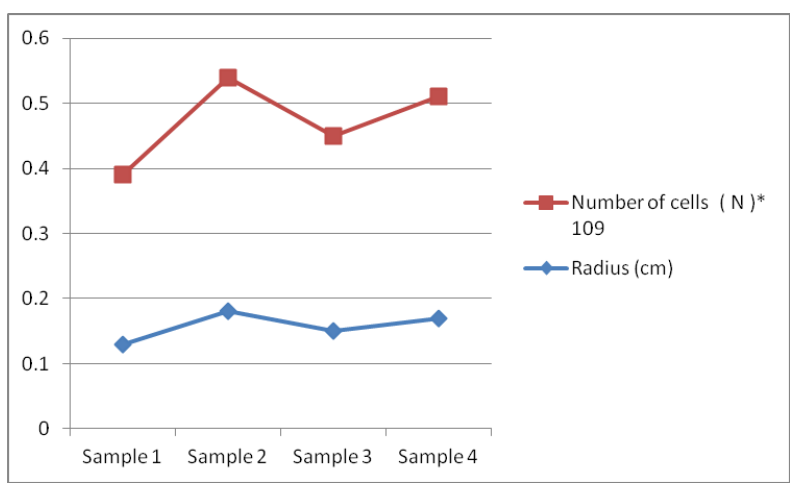

Fig.10 Radius of the tumour Vs number of cells

The resolute image is analyzed with quality parameters results evincing acceptable quality image .The proposed "OHC" approach proved as a refined and respectable method to estimate the absolute area of the tumor and its volume. The number of tumor cells are estimated w.r.t its radius. Reckoning the tumor area, volume, and number of aberrant tissue cells will play a vital role to treat the tumors. 
Subsequently the location of the tumor may be determined in addition with the size i.e. area of the tumor. Precisely the quantitative analysis of the tumor will play a vital role for applying the radiation to annihilate the tumor cells. The Proposed method exhibits reliable values when compared with existing approaches. Estimating the absolute tumor area, it's radius it's volume and number of tumor cells results a supporting tool to the physician for treating the tumors. The advances in tumor diagnosis can increase the survival of tumor patients. In future the Tumor exact location is to be calculated for therapeutic application.

\section{CONCLUSION}

Median filter proved as a reliable filter to remove the noise from the MR images. DWT + SWT itself is more suggestible for Enhancement of the MR images. The proposed algorithm produced favorable results to estimate the area and volume of the tumor accurately. Estimating the number of tumor cells will give supportive tool to the physician while pumping the medicine to kill the tumor cells.

In future, the work is proposed to extend for metastasis brain tumors.

\section{ACKNOWLEDGEMENT}

Thanks to Dr. Krupasagar kalapala and Dr. Srujana Ulavapati for their constant support and guidance throughout this work and Dr. Anil for providing the necessary MRI images for processing.

\section{REFERENCES}

[1] Govind N Sarage, Dr sagar Jambhorkar," Magnetic Resonance Image Denoising Using Laplacian Filtering Technioque" International Journal of Advanced Ressearch in computer science and software Engineering Vo 2, 2012.

[2] Rajanand Gupta, “ Algorithm for Image Processing using improved median filter and comparision of Mean, Median and Improved Median Filter" International Journal of soft computing and engineering,Vol1, 2011

[3] Sunaya U.Shirodkar," Image Resolution Enhancement using various wavelet Transfors", International journal of advances in science Engineering and Technology, Voll, 2014.

[4] V.C.MIAINDARGI, A.P.MANE "Decimated and UnDecimated Wavelet Transforms based Image Enhancement"International journal of industrial Electrical, Electronics, Control and Robotics, Vol.03, Issue. 05,2013

[5] B.Sivakumar, S.Nagaraj "Descrete and stationary wavelet decomposition for image Resolution Enhancement" International Journal of Engineering trends and Technology, Volume4,2013

[6] Mr.G.M.Khaire, R.P.Shelkikar," Resolution Enhancement of images with interpolation and DWT SWT Wavelet domain components" , International Journal of Application or Innovation in Engineering and Management, Vol2, 2013.
[7] S. Ray, R.H. Turi, "Determination of number of clusters in K-means clustering and application in colthe image segmentation”, Proc. 4th ICAPRDT, pp. 137-143, 1999.

[8] P. Dhanalakshmi, T. Kanimozhi," Automatic segmentation of brain tumor using $\mathrm{k}$ means clustering and its area calculation" International Journal of advanced electrical and Electronics Engineering, volume 2 , issue 2,2013

[9] Rohit S Khabade ,M.S.Gaikwad ," Segmentation of Brain tumor and its area calculation in brain MR images using K mean Clustering and fuzzy C mean algorithm", International journal of Computer science and Technology, Vol 4No.05, May 2013.

[10] Chu-Xiaoli,Zhu-ying,Shi-Zuntao,Song-JiQing "Method of Image Segmentation based on Fuzzy C-Means Clustering and Artificial Fish Awarm Algorithm"Intelligent Computing and Integrated Systems (ICISS) International Conference,22-24 Oct 2010

[11] Gabriela. Erban, Grigoreta sofia Moldovan "A Comparison of Clustering techniques in aspect mining" Studia niv.Babe._BoliaI,Informatica, Vo L1,Number 1,2006 .

[12] Kehar Singh,Dimple Malik,Naveen Sharma, "Evolving Limitations in K-Means Algorithm in data mining and their removal" International Journal of Computational Engineering \& Management, Vol. 12, April 2011.

[13] M. Gowthami Reddy,D. Gowri Sankar Reddy, "A novel scheme for brain tumor detection and area calculation using pillar K-Means" International Journal of Recent Advances in Engineering \& Technology, Volume-1, Issue - 2, 2013

\section{AUTHOR PROFILE}

K.Vidyasagar Received the B.Tech degree in Instrument Technology from Andhra University College of Engineering Visakhapatnam, the M.E from P.S.G.Tech Coimbator. He is now a research scholar under the guidance of Dr.A. Bhujangarao,Andhra University. His current research interests include image processing in biomedical instrumentation and related embedded systems.

Dr. A.Bhujangarao received Ph.D in Instrumentation from Andhra University. who is currently professor and HOD, at Department of InstrumentTechnology AndhraUniversity college of Engineering, Visakhapatnam, he is specialized in Instrumentation and acoustic remote sensing Instrumentation, he published 20 journal papers and 18 conference papers. His current research interest is on Bio Medical instrumentation and process control instrumentation.

T.Madhu received Ph.D., from Osmania University, Hyderabad. He is now working as Principal, Swarnandhra Institute of Engineering \& Technology, Seetharampuram, Narasapur. His current research interests include GPS data analysis \& Modeling, speech \& image processing and RF design 